\title{
BMJ Open A protocol for an exploratory phase I mixed-methods study of enhanced integrated care for care home residents with advanced dementia: the Compassion Intervention
}

Margaret Elliott, Jane Harrington, Kirsten Moore, Sarah Davis, Nuriye Kupeli, Victoria Vickerstaff, Anna Gola, Bridget Candy, Elizabeth L Sampson, Louise Jones

To cite: Elliott M,

Harrington J, Moore K, et al. A protocol for an exploratory phase I mixed-methods study of enhanced integrated care for care home residents with advanced dementia: the Compassion Intervention. BMJ Open 2014;4:e005661. doi:10.1136/bmjopen-2014005661

- Prepublication history for this paper is available online. To view these files please visit the journal online (http://dx.doi.org/10.1136/ bmjopen-2014-005661).

Received 8 May 2014 Accepted 2 June 2014

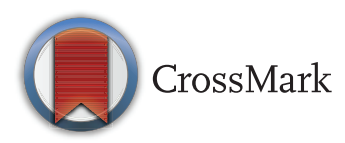

Marie Curie Palliative Care Research Unit, Division of Psychiatry, University College London Medical School, London, UK

Correspondence to Dr Elizabeth L Sampson; e.sampson@ucl.ac.uk

\section{ABSTRACT}

Introduction: In the UK approximately 700000 people are living with, and a third of people aged over 65 will die with, dementia. People with dementia may receive poor quality care towards the end of life. We applied a realist approach and used mixed methods to develop a complex intervention to improve care for people with advanced dementia and their family carers. Consensus on intervention content was achieved using the RAND UCLA appropriateness method and mapped to sociological theories of process and impact. Core components are: (1) facilitation of integrated care, (2) education, training and support, (3) investment from commissioners and care providers. We present the protocol for an exploratory phase I study to implement components 1 and 2 in order to understand how the intervention operates in practice and to assess feasibility and acceptability.

Methods and analysis: An 'Interdisciplinary Care Leader (ICL)' will work within two care homes, alongside staff and associated professionals to facilitate service integration, encourage structured needs assessment, develop the use of personal and advance care plans and support staff training. We will use qualitative and quantitative methods to collect data for a range of outcome and process measures to detect effects on individual residents, family carers, care home staff, the intervention team, the interdisciplinary team and wider systems. Analysis will include descriptive statistics summarising process and care home level data, individual demographic and clinical characteristics and data on symptom burden, clinical events and quality of care. Qualitative data will be explored using thematic analysis. Findings will inform a future phase II trial.

Ethics and dissemination: Ethical approval was granted (REC reference 14/L0/0370). We shall publish findings at conferences, in peer-reviewed journals, on the Marie Curie Cancer Care website and prepare reports for dissemination by organisations involved with end-of-life care and dementia.

\section{Strengths and limitations of this study}

- The intervention has been developed within a realistic framework using mixed methods and undertaking data synthesis from a wide range of sources and locations; the core components have been mapped to sociological theory of impact and process to enhance its validity and the likelihood of it being feasible and acceptable in practice.

- The collection of a wide range of process and outcome data using mixed methods and implementation in two sites will increase our understanding of enablers and barriers to the intervention, the effects of context and local organisational pressures, which components might be generalisable across care settings and which might be particularly sensitive to local factors.

- The sample size is small and it will not be possible to report statistically significant outcomes or definitive results; this is an exploratory study in which the main aims are to assess feasibility, acceptability and economic cost, in order to refine the intervention before embarking on a phase II trial.

\section{INTRODUCTION}

The number of people worldwide living and dying with dementia is increasing. ${ }^{12}$ In the UK, approximately 700000 people are living with dementia $^{3}$ and approximately one-third of people aged over 65 will die with dementia. ${ }^{4}$ However, reports suggest that people with dementia may receive poor quality care towards the end of life; care may fail to meet their physical and/or emotional needs and may not consider the needs of family carers. ${ }^{5}{ }^{6}$ Symptoms experienced in the last year of life by people with advanced dementia, such as pain, breathing difficulties, pressure sores, impaired 
appetite and difficulty swallowing, are comparable to those experienced by people with advanced cancer. ${ }^{78}$ Referral to palliative care services for people with dementia is rare and less than $1 \%$ of hospice patients in Europe have a neurological diagnosis. ${ }^{9}$ However, for many people with advanced dementia their symptoms and care needs could be managed by generalist providers. We suggest that good care requires a broad, but cost effective, palliative approach, which is person-centered and tailored to meet individual needs. Our work responds to UK government initiatives for care in dementia and at the end of life. ${ }^{10-12}$

The Compassion Programme (figure 1) is a 3-year National Institute for Health Research (NIHR) portfolio programme of research funded by Marie Curie Cancer Care. We have used mixed qualitative and quantitative evidence and a realist approach to develop a complex intervention, 'Compassion', designed to improve care for people with advanced dementia approaching the end of life. ${ }^{13}$ In this paper we present the protocol for an exploratory phase I study of implementation of the Compassion Intervention in two UK healthcare economies (Clinical Commissioning Groups).

\section{Intervention development}

'Compassion' is a complex intervention that delivers a model of enhanced integrated care; it is described in detail in a written manual. It has been developed through an iterative process bringing together data from a range of sources and locations.

1. A literature review.

2. A review of UK health and social care policy.

3. Workshops across the UK with health and social care professionals.

4. A workshop with patients with early dementia and a workshop with family carers.

5. Qualitative interviews with health and social care professionals.
6. Quantitative data from people with advanced dementia and their carers participating in a cohort study of clinical need and carer burden.

Consensus on intervention content was achieved using the RAND UCLA appropriateness $\operatorname{method}^{14}$ with participants in workshops across all four countries of the UK. To enable a deeper understanding of how the intervention may exert its effects, individual components of the intervention have been mapped to sociological theories of process and impact. ${ }^{15} 16$ Details of these elements of the research will be reported separately.

We describe three overarching core components of the Compassion Intervention:

1. Facilitation of integrated care;

2. Education, training and support;

3. Investment from commissioners and care providers.

In this exploratory study, we implement components 1 and 2, which, over time, may influence component 3. Each core component contains a number of subcomponents which we shall refine further using results from this study.

\section{Intervention structure}

The Compassion Intervention will be delivered by what we describe as an 'Interdisciplinary Care Leader (ICL)' who has experience of working with people with dementia in care home settings. The ICL will be based within participating care homes and will work alongside care home staff and associated health and social care professionals currently providing routine care. The ICL will receive training in the Compassion Intervention and supervision from the programme team to check adherence to the Compassion manual. Further training will cover standard procedures of clinical and information governance, safeguarding of vulnerable adults and the Mental Capacity Act. The ICL will seek to:

- Understand which professionals are providing care, care pathways and services currently available;
Figure 1 Work-streams of the Compassion Programme.

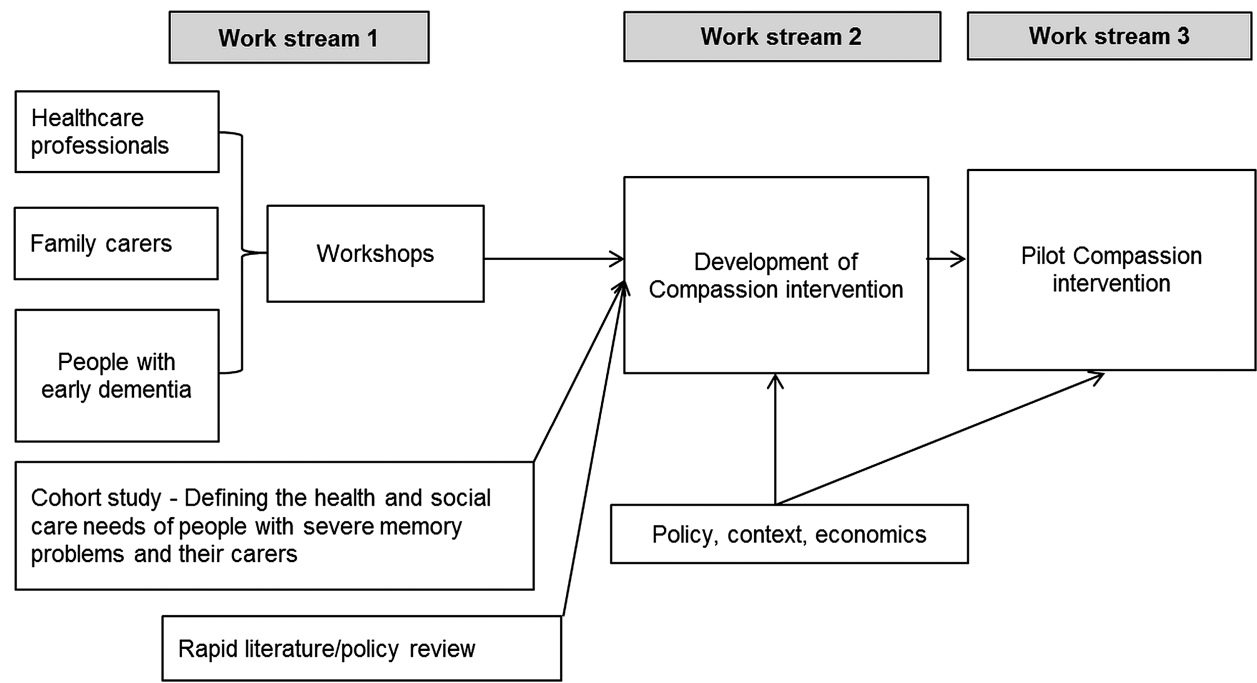


- Develop joint working to enable integration of available services;

- Encourage a proactive structured assessment of the needs of residents with advanced dementia;

- Facilitate understanding and use of personal and advance care plans;

- Provide support to front-line staff to enable them to avoid unnecessary place of care transfers;

- Identify and support the training needs of care home staff;

- Foster a culture of respect, dignity and quality of care for all residents;

- Meet with family carers to ensure their needs and wishes are understood;

- Act as a central resource for health and social care professionals, care home staff and family carers.

Figure 2 outlines the key steps of the intervention pathway.

The Core Team: The ICL will meet weekly with the existing core team responsible for the care of residents. This team will include the clinical lead professional (GP, geriatrician or old age psychiatrist) and a member of the care home staff (care home manager or floor/unit manager).

The Wider Interdisciplinary Team: Monthly meetings will be held with the wider interdisciplinary team, which will include representatives from specialist palliative care, old age psychiatry, care of the elderly, social care.
Educational and training needs: These will be addressed through shared working, use of online resources and formal topic-based teaching if required. Training will link to nationally agreed core competencies. ${ }^{17}$

\section{Study aims and objectives}

The study aims are:

1. To understand how the Compassion Intervention operates in practice, in two care homes, in two different health and social care economies (Clinical Commissioning Groups);

2. To collect process and outcome data on feasibility, acceptability and economic costs in order to inform the planning of a future phase II trial.

Our objectives are:

- To understand whether the intervention is feasible in the setting;

- To determine whether it is acceptable to staff and family carers;

- To understand the facilitators and barriers to implementation;

- To evaluate a range of potential process and outcome measures;

- To describe in detail the costs of delivery.

\section{METHODS}

\section{Study design}

The study is a naturalistic phase I exploratory study of the implementation of the Compassion Intervention. We
Figure 2 Compassion Intervention flow chart (ICL, Interdisciplinary Care Leader).

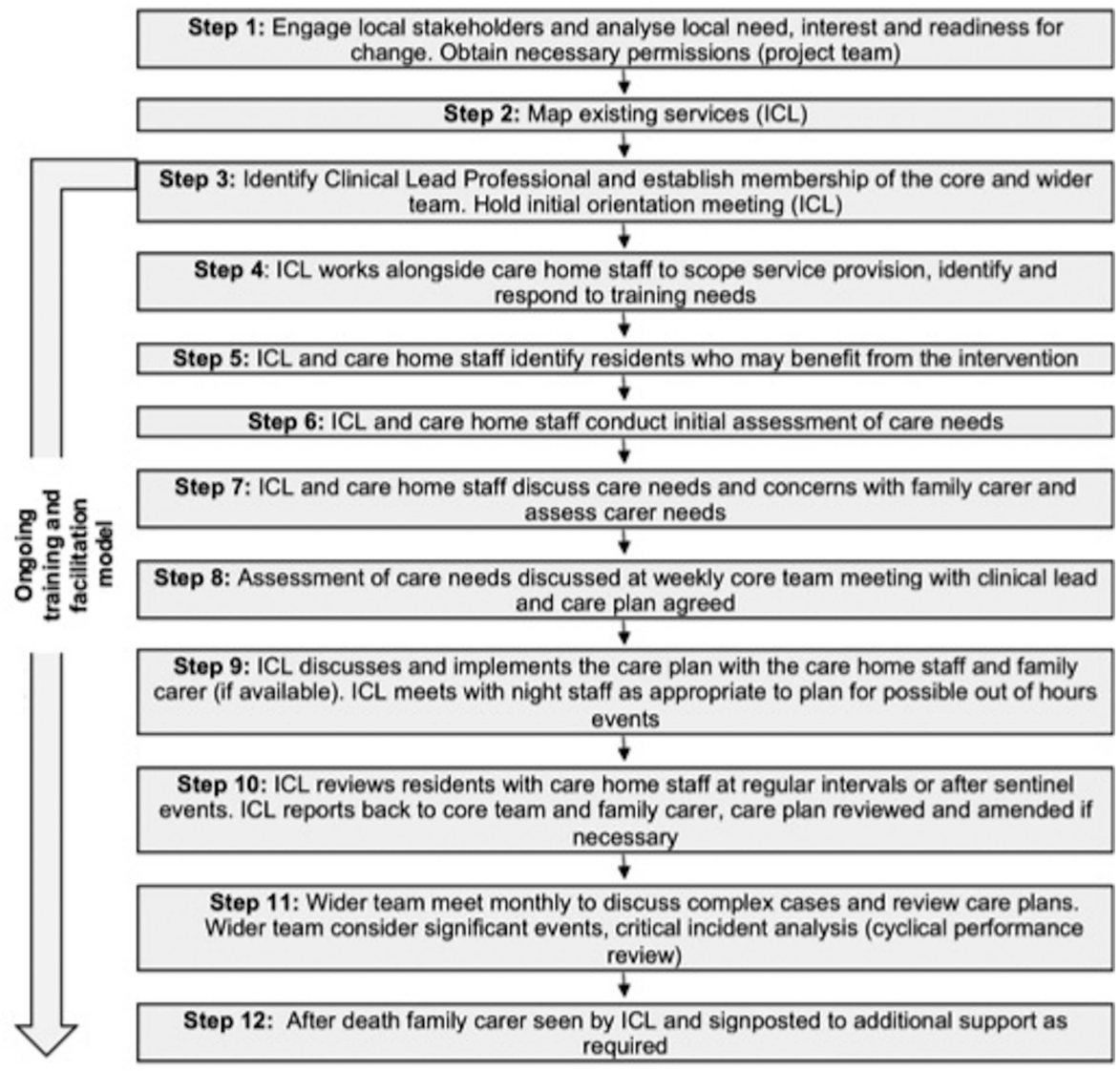


expect that the intervention will operate at a number of levels and therefore we will collect data on a range of outcome and process measures to detect effects on individual residents, family carers, care home staff, the intervention team, the interdisciplinary team and wider systems. Anonymised process and outcome data will be collected by the ICL, with the support of the care home manager, for all residents included within the intervention and for the care home as a whole. In addition we shall seek consent to collect individual-level outcome data from all those connected with the intervention to allow a more detailed evaluation; this work will be completed by the research team, independent of the ICL, to ensure methodological rigour.

\section{Setting}

Service providers within two Clinical Commissioning Groups (CCGs) within greater London, UK, at different levels of service development, have agreed to take part in the pilot study. We shall work within one care home in each CCG and with local primary and secondary health and social care providers. Written consent for the care homes to participate in the Compassion Intervention, and for process data on study outcomes to be collected, has been sought from the manager and owner.

\section{Participants}

Implementation of the intervention is at the level of the care home and all residents who fit the inclusion criteria will receive the enhanced service. Inclusion criteria are based on an existing model of enhanced care which has been quoted as an example of UK best practice. ${ }^{18}$

Inclusion criteria for people with dementia

1. Aged over 65 years.

2. Memory problems indicating a clinical diagnosis of Diagnostic and Statistical Manual of Mental Disorders Fourth Edition (DSM-IV) dementia. ${ }^{19}$

3. Moderately severe or severe dementia as classified on the Functional Assessment Staging scale (FAST) grade $6 \mathrm{a}$ and above. ${ }^{20}$ (At stage $6 \mathrm{a}+$ people with dementia have difficulty dressing and bathing, and may be incontinent. By stage $7 \mathrm{a}+$ they are doubly incontinent with limited speech, and mobility is reduced or lost.)

Plus at least one of the following:

- Recurrent infections or fevers, pain, falls, pressure ulcers, significant weight loss or physical frailty;

- Severe, persistent distress (mental or physical) that is not easily amenable to treatment;

- Another condition (eg, cancer) whose coexistence with dementia means that intrusive treatments may not be appropriate.

Exclusion criteria for people with dementia:

1. Residents who indicate either verbally or non-verbally they do not wish to participate.

2. Residents who are moribund, in a coma or who present clinical concerns that preclude participation.

\section{Sample size}

This is an exploratory study and therefore numbers of participants are chosen on pragmatic grounds. We will include as many eligible residents as possible within the intervention. For individual outcome data collection across the two study sites we aim to recruit up to 30 residents with advanced dementia, 15 family carers and 10 health and social care professionals.

\section{Implementation timetable}

We shall introduce the Compassion Intervention at site 1 (an inner city CCG) and collect data over 6 months. We shall use learning gathered to make any further adjustments to the intervention manual and to inform the implementation process at site 2 (a suburban CCG) which will commence $6-8$ weeks after site 1 is started.

Implementation of the intervention will follow four phases: orientation and insight (scoping); acceptance and adoption of the intervention; change, during which new ways of working occur; maintenance and sustainability. ${ }^{15}$

\section{Collection of process and care home-level outcome data}

Process data and care home-level outcome data will be collected by the ICL and care home manager. This will include the number of:

- Residents seen by the ICL (new and review);

- Contacts with family carers (by phone and face to face);

- Individual care plans made by the enhanced care team;

- Residents with a pain management plan;

- Residents with a surrogate decision maker recorded;

- Residents with resuscitation status recorded;

- Residents with preferred place of death recorded;

- Cases discussed at the interdisciplinary team meetings;

- Referral to specialists services, for example, dietician, tissue viability nurse;

- 999 (emergency) calls and acute hospital admissions;

- $11124 \mathrm{~h}$ NHS medical assistance calls and GP out of hours visits;

- Deaths (total), number occurring in care home and preferred place of care;

- Complaints and compliments regarding care of residents.

The ICL will identify the education and training needs of care home staff and document how these needs are being addressed. The ICL will also keep a reflective diary (ensuring anonymity and confidentiality) recording his/her experiences of implementing the intervention, care home dynamics, interactions with core and wider interdisciplinary teams and any changes in care being observed. 
Collection of individual outcome data from residents with advanced dementia

Data on individual outcomes will be collected by the research team and will only be collected from participants for whom individual signed consent/assent has been obtained.

Recruitment and consent: Residents with advanced dementia may not have capacity to give informed consent; therefore our procedures have been developed to comply with capacity legislation governing England and Wales (Mental Capacity Act 2005, Sections 30-34) as shown in figure 3 . The care home manager will assess the capacity of residents to give informed consent; if the resident has capacity they will be asked if they are willing to discuss the study with a member of the research team. When capacity is not present the care home manager will identify the next

\section{Potential participants from care homes}

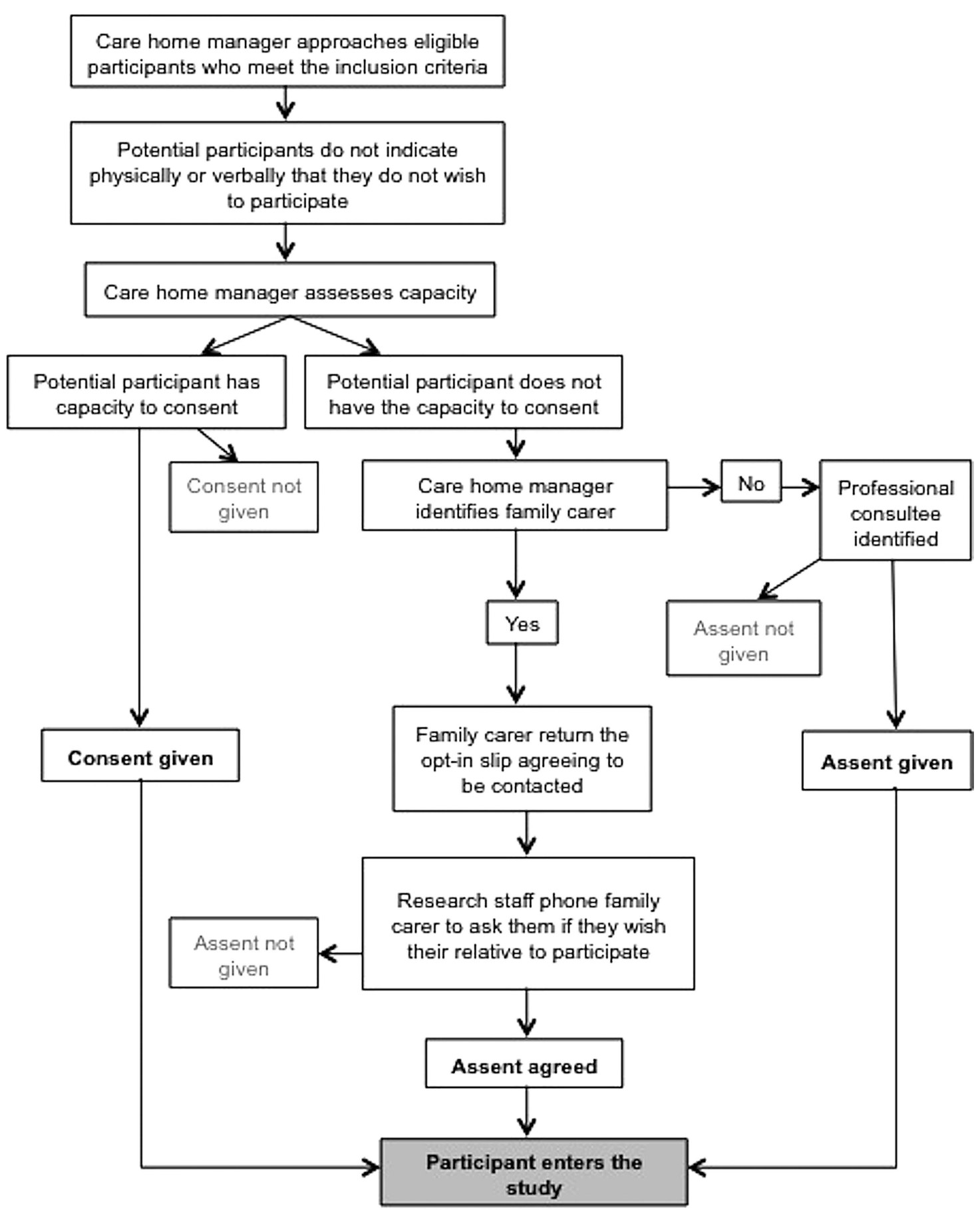

Figure 3 Summary of resident consent procedures for the collection of individual outcome data. 
of kin, or someone close to the resident (who does not receive remuneration for this role) to act as a 'personal consultee'. The personal consultee will be provided with written information about the study, including a reply slip indicating agreement to be contacted by the research team. If permission is granted, the research team will discuss the study in more detail and if agreement is reached, obtain signed assent. If a personal consultee cannot be identified the research team will contact a professional consultee, defined as a senior experienced health or social care worker who is not directly involved in the research or direct clinical care of the patient. Professional consultees will follow a structured procedure based on the 2005 Mental Capacity act to give or decline written assent for the resident with dementia to participate in the study.

Outcome data to be collected: Participating residents will be seen at baseline study entry and then monthly, for a maximum of 6 months or until death. Demographic information will be collected at baseline. The presence of advance directives, advance care plans and specific requests regarding hospitalisation and resuscitation will be documented at baseline and during the course of the intervention. Prescribed medications, the number and reason for acute hospital admissions, 'burdensome interventions' and 'sentinel events', defined as 'new medical conditions that have the potential to lead to a significant change in health status and a shift in the goals of care', ${ }^{7}$ will be recorded monthly. All other outcome measures to be collected are shown in table 1 .

\section{Collection of individual outcome data from family carers}

Family carers will only be approached when consent/ assent has already been given for individual outcome data to be collected from the resident with dementia. The recruitment of resident/family carer dyads will enable us to link the experiences of people with advanced dementia and their family carers.

Inclusion criteria for family carers

1. If the resident does not have capacity this is the main family carer or 'key decision maker' as identified by the care home manager. If the resident does have capacity they will nominate their family carer.

2. English language sufficient to complete the study ratings.

Exclusion criteria for family carers

1. Where there are clinical concerns that may preclude them being approached.

2. Aged 16 and under.

3. If during the study the family carer becomes unavailable or unable to give consent we will withdraw them from the study.

\section{Recruitment and consent}

The care home manager will provide family carers with written information about the study, including a reply slip indicating agreement to be contacted by the research team. If permission is granted the research team will discuss the study in more detail and, if agreement is reached, written consent will be obtained.

\section{Outcome data to be collected}

Family carer data collection will occur at baseline study entry, within 14 days of the initial resident assessment, and then monthly. If the resident dies, with the carer's further agreement, outcome data will be collected at 2 months postbereavement. Demographic data including

Table 1 Individual outcome measures for care home residents receiving the Compassion Intervention

\begin{tabular}{|c|c|c|}
\hline Measure/author & Purpose & $\begin{array}{l}\text { Time of } \\
\text { assessment }\end{array}$ \\
\hline Charlson comorbidity index ${ }^{21}$ & Calculates severity of chronic comorbidities & Baseline \\
\hline Functional Assessment Staging Scale (FAST) ${ }^{20}$ & $\begin{array}{l}\text { Describes a continuum of } 7 \text { stages of } \\
\text { functional impairment }\end{array}$ & Baseline \\
\hline Waterlow Scale 22 & Risk for developing pressure sores & Baseline/monthly \\
\hline Bedford Alzheimer Nursing Scale (BANS) ${ }^{23}$ & $\begin{array}{l}\text { Used to stage levels of severe memory } \\
\text { impairment in dementia }\end{array}$ & Baseline/monthly \\
\hline The Neuropsychiatric Inventory (NPI) ${ }^{24}$ & $\begin{array}{l}\text { Assesses behavioural and psychological } \\
\text { symptoms in dementia }\end{array}$ & Baseline/monthly \\
\hline Cohen Mansfield Agitation Inventory (CMAI) ${ }^{25}$ & $\begin{array}{l}\text { Measures a range of agitated and potentially } \\
\text { challenging behaviours }\end{array}$ & Baseline/monthly \\
\hline $\begin{array}{l}\text { Pain Assessment in Advanced Dementia } \\
\text { (PAINAD) }{ }^{26}\end{array}$ & $\begin{array}{l}\text { Observational pain tool used to measures pain } \\
\text { during care tasks and at rest }\end{array}$ & Baseline/monthly \\
\hline $\begin{array}{l}\text { Symptom Management at the End of Life in } \\
\text { Dementia Scale (SM-EOLD) }\end{array}$ & Assesses comfort and pain in the prior 30 days & Baseline/monthly \\
\hline $\begin{array}{l}\text { Comfort Assessment in Dying with Dementia Scale } \\
\text { at the End of Life CAD-EOLD) })^{27}\end{array}$ & Comfort at the end of life & Postdeath \\
\hline $\begin{array}{l}\text { Quality of Life in Late Stage Dementia Scale } \\
(\text { QUALID) })^{28}\end{array}$ & Quality of life in the prior week & Baseline/monthly \\
\hline $\begin{array}{l}\text { The Resource Utilisation in Dementia Questionnaire } \\
\text { (RUD-LITE) }\end{array}$ & $\begin{array}{l}\text { Formal and informal health and social care } \\
\text { resource use }\end{array}$ & $\begin{array}{l}\text { Baseline/monthly/ } \\
\text { postdeath }\end{array}$ \\
\hline
\end{tabular}


Table 2 Individual outcome measures for family carers

\begin{tabular}{|c|c|c|}
\hline Measure/author & Purpose & Time of assessment \\
\hline Zarit Burden Interview ${ }^{30}$ & $\begin{array}{l}\text { Reflects feelings about caring, measures } \\
\text { carer burden }\end{array}$ & Baseline/monthly \\
\hline $\begin{array}{l}\text { Hospital Anxiety and Depression Scale } \\
(\text { HADS })^{31}\end{array}$ & $\begin{array}{l}\text { Assesses symptoms of anxiety and } \\
\text { depression }\end{array}$ & $\begin{array}{l}\text { Baseline/monthly and } \\
2 \text { months postdeath }\end{array}$ \\
\hline $\begin{array}{l}\text { Satisfaction with Care at the End of Life } \\
(\text { SWC-EOLD })^{27}\end{array}$ & $\begin{array}{l}\text { Assesses the carer's level of satisfaction } \\
\text { with care in advanced dementia }\end{array}$ & $\begin{array}{l}\text { Baseline/monthly and } \\
2 \text { months postdeath }\end{array}$ \\
\hline $\begin{array}{l}\text { Comfort Assessment in Dying with Dementia } \\
\text { Scale at the End of Life (CAD-EOLD) }\end{array}$ & $\begin{array}{l}\text { Assesses the carer's perception of } \\
\text { symptoms and comfort at the end of life }\end{array}$ & 2 months postdeath \\
\hline$E Q-5 D-5 L^{32}$ & $\begin{array}{l}\text { Used for calculation of quality-adjusted life } \\
\text { years }\end{array}$ & Baseline/monthly \\
\hline
\end{tabular}

age, gender, employment, relationship to the resident, the number of years spent caring and any other caring responsibilities will be collected at baseline. All other outcome measures to be collected are shown in table 2 .

To gain a deeper understanding of their experience of caring for someone with advanced dementia, and of any impact the Compassion Intervention may have had, qualitative interviews will be offered to all participating family carers at the end of the study. If the resident participant dies during the study, interviews will be offered to family carers 2 months postbereavement. At this point, the family carer is more likely to feel ready to consider their loss with sufficient recall of events ${ }^{33} 34$ and to be willing to reflect on their experiences. ${ }^{35}$

\section{Interviews with care home staff, health and social care professionals}

Care home staff and associated health and social care professionals will be offered the opportunity to participate in qualitative interviews at the end of the study.

Recruitment and consent: The care home manager will identify care home staff and visiting professionals who have been involved in the intervention and ask if they are interested in participating in the research study. If permission is granted, the research team will contact individual staff members to provide further information and, if agreement is reached, written consent will be obtained.

Data to be collected: Semistructured interviews will explore the participant's experience of the Compassion Intervention and caring for people with advanced dementia. All interviews will follow a topic guide, last a maximum of $1 \mathrm{~h}$, be audiotaped and transcribed verbatim (anonymised); participants will be offered the opportunity to review transcripts to ensure accuracy.

\section{Potential risks during individual outcome data collection}

All outcome measures for residents with dementia involve the collection of observational data with no additional burden or discomfort to the participant; many of these measures are part of good end-of-life care. ${ }^{36}$ However, if the resident does become upset or uncomfortable the assessment will be stopped immediately and the circumstances reported to care home staff and/or the family carer. If we discover issues of malpractice, maltreatment or serious neglect, to the degree that local authority's safeguarding procedures are triggered, we will initiate this process.

Our research may touch on sensitive issues for family carers; however, the research team is experienced in conducting interviews with relatives of patients with malignant and non-malignant conditions, including advanced dementia, during the final stages of illness and into bereavement. ${ }^{35} 37^{38}$ If the family carer does become distressed, the researcher will stop the assessment and ask the participant if they would like to take a break or bring the assessment to a close. It is natural that family carers may feel emotional when talking about their relative; however, if the assessment suggests clinical depression or anxiety they will be given information regarding support agencies or directed to contact their general practitioner.

\section{Data management}

Data will be stored securely and entered into a passwordprotected anonymised database by the research team. To ensure methodological rigour the research team, but not the ICL, will have access to the data.

\section{Quantitative analysis}

We shall report descriptive statistics summarising process and care home-level data. We shall describe individual demographic and clinical characteristics of participating residents and family carers. Data on symptom burden, clinical events, interventions, sentinel events and quality of care will be presented and summarised using means and SDs or alternatives in the case of non-normally distributed data. Data will be compared with results from the Compassion cohort study to begin to understand the possible impact of the Compassion Intervention.

\section{Qualitative analysis}

We shall use the qualitative software programme Atlas-ti for coding, management and retrieval of data. Interviews will be transcribed verbatim and analysed using thematic content analysis, ${ }^{39}$ working to guidelines 
and quality frameworks. ${ }^{40}$ Throughout the analysis researchers will use memos and reflective diaries to engage with the data and refine emergent themes through an iterative and inductive process. Data triangulation will be achieved by comparing interview data from family carers, care home staff and interdisciplinary team members to explore the intervention from different perspectives.

\section{Health economics}

A microeconomic approach will be used to estimate the overall costs of the intervention delivery and of its individual components, such as time spent, personnel involvement and medication use. Areas of interest, such as whether prescribing of medication increases or decreases, will be explored. We will obtain point estimates and corresponding CIs for these variables.

\section{DISCUSSION}

The Compassion Intervention aims to enhance care by facilitating best practice and by offering additional but cost-effective mechanisms to enable existing systems to work in a more integrated way. It aims to build on what is already working well, and adjust what may need further development to maximise benefits for people with dementia, their family and paid carers. Our methods are based on the recommendations of the Medical Research Council framework for the development and testing of complex healthcare interventions, ${ }^{41}$ and key publications in the field of implementation science. ${ }^{15} 1642$ One recognised challenge for healthcare innovations is the maintenance of change once the implementation team departs. Sustainability depends on the success of the team in embedding change into everyday practice, on a positive experience by those involved and on the degree of benefit observed or perceived. Recent thinking suggests that the essence of the intervention might be absorbed by a range of team members and that each may take forward different components according to what is possible and relevant for them personally. This concept of 'dynamic sustainability' may therefore enable teams to work with their new knowledge in ways that may be potentially more effective, and this may enhance a true cultural shift towards the provision of better care. ${ }^{43}$

Strengths of this study are that the intervention has been developed within a realistic framework, ${ }^{44}$ bringing together qualitative and quantitative data from a wide range of sources, including directly from people with dementia and family and paid carers. Evidence suggests that interventions which take in to account the views of participants during their development are more likely to be effective in practice. ${ }^{45}$ Furthermore, understanding the underlying theories through which Compassion might operate enhances its validity and the likelihood of it being feasible and acceptable in practice; we anticipate that the rigour of our approach will support the
ICL when engaging professionals in its delivery. Finally, implementation in two sites will increase our understanding of the enablers and barriers to the intervention, the effects of context and local organisational pressures, which components might be generalisable across care settings and which might be particularly sensitive to local factors.

The study does have a number of limitations. First, the sample size is small and it will not be possible to derive statistically significant or definitive results; second, although implementation is taking place in two sites these are both within London and this may limit the generalisability of findings; third, outcomes may be influenced by factors external to the intervention itself, such as other local and national initiatives to improve end-of-life care. However, this is an exploratory study in which the main aims are to assess feasibility, acceptability and describe economic costs.

Data and understanding emerging from this study will be used to refine the Compassion Intervention and inform further research. Initial phase II work led by members of this research team will form part of the MARQUE programme ${ }^{46}$ funded by ESCR/NIHR as part of the UK Prime Minister's Dementia Challenge 2012.

Acknowledgements The authors acknowledge the contribution to this work by former colleagues, Sharon Scott, Kathryn Lord and Jeff Round who worked on earlier stages of this programme of research.

Contributors LJ and ELS devised the programme of research and secured the funding. All authors contributed to the writing of intervention protocol. ME, $\mathrm{JH}$, ELS and LJ drafted this manuscript. All authors critically revised the manuscript for important intellectual content. All authors read and approved the final manuscript.

Funding The Compassion Programme is funded by Marie Curie Cancer Care (grant ref. MCCC-FPR-11-U) through a process administered in partnership with Cancer Research UK.

\section{Competing interests None.}

Ethics approval Ethical approval has been granted for this exploratory intervention study by the National Research Ethics Service (NRES) Committee, London-Camden and Islington REC reference 14/LO/0370.

Provenance and peer review Not commissioned; internally peer reviewed.

Open Access This is an Open Access article distributed in accordance with the Creative Commons Attribution Non Commercial (CC BY-NC 4.0) license, which permits others to distribute, remix, adapt, build upon this work noncommercially, and license their derivative works on different terms, provided the original work is properly cited and the use is non-commercial. See: http:// creativecommons.org/licenses/by-nc/4.0/

\section{REFERENCES}

1. Ferri CP, Prince M, Brayne C, et al. Global prevalence of dementia: a Delphi consensus study. Lancet 2005;366:2112-17.

2. Brookmeyer R, Johnson E, Ziegler-Graham K, et al. Forecasting the global burden of Alzheimer's disease. Alzheimers Dement 2007;3:186-91.

3. Matthews F, Arthur A, Barnes LE, et al. A two decade comparison of prevalence of dementia in individuals aged 65 years and older from three geographical areas of England: results of the Cognitive Function and Ageing Study I and II. Lancet 2013;382:1405-12.

4. Brayne C, Gao L, Dewey M, et al. Dementia before death in ageing societies - the promise of prevention and the reality. PLOS Med 2006;10:e397.

5. Vandervoort A, Van den Block L, van der Steen J, et al. Nursing home residents dying with dementia in Flanders, Belgium: a 
nationwide post mortem study on clinical characteristics and quality of dying. J Am Med Dir Assoc 2013;14:485-92.

6. Lawrence V, Samsi K, Murray J, et al. Dying well with dementia: qualitative examination of end-of-lifecare. $\mathrm{Br} J$ Psychiatry 2011;199:417-22.

7. Mitchell SL, Teno JM, Kieley DK, et al. The clinical course of advanced dementia. N Engl J Med 2009;361:1529-38.

8. Hall $P$, Schroder $C$, Weaver L. The last 48 hours of life in long-term care: a focused chart audit. J Am Geriatr Soc 2002;50:501-6.

9. Davies E, Higginson IJ. Better palliative care for older people. World Health Organisation Europe, 2004. http://www.euro.who.int/_data/ assets/pdf file/0009/98235/E82933.pdf (accessed Apr 2014).

10. Department of Health. End of life care strategy: promoting high quality care for all adults at the end of life. London: Department of Health, 2008. https://www.gov.uk/government/publications/end-oflife-care-strategy-promoting-high-quality-care-for-adults-at-the-endof-their-life (accessed May 2014).

11. Department of Health. Living well with Dementia: a national dementia strategy. London: Department of Health, 2009. https:// www.gov.uk/government/publications/living-well-with-dementiaa-national-dementia-strategy (accessed May 2014).

12. Department of Health. Prime minister's challenge on dementia. Delivering major improvements in dementia care and research by 2015. 2012. https://www.gov.uk/government/uploads/system/uploads/ attachment data/file/215101/dh_133176.pdf (accessed May 2014).

13. Jones L, Harrington J, Scott S, et al. CoMPASs: IOn programme (Care of Memory Problems in Advanced Stages of dementia: Improving Our Knowledge): protocol for a mixed methods study. BMJ Open 2012;2:e002265.

14. Fitch K, Bernstein SJ, Aguilar MD, et al. The RAND/UCLA appropriateness method user's manual. Santa Monica: RAND, 2001.

15. Grol RP, Bosch MC, Hulscher ME, et al. Planning and studying improvement in patient care: the use of theoretical perspectives. Milbank Q 2007;85:93-138.

16. Ferlie EB, Shortell SM. Improving the quality of health care in the United Kingdom and the United States: a framework for change. Milbank Q 2001;79:281-315

17. Common Core Competencies and Principles: a guide for health and social care workers with adults at the end of life. http://www. skillsforcare.org.uk/Skills/End-of-life-care/End-of-life-care.aspx (accessed Apr 2014)

18. Sonola L, Thiel V, Goodwin N, et al. Oxleas advanced dementia service. Supporting carers and building resilience. The Kings Fund, 2013. http:// www.kingsfund.org.uk/publications/oxleas-advanced-dementia-service (accessed May 2014)

19. American Psychiatric Association. Diagnostic and Statistical Manual of Mental Disorders (DSM-1V). Washington, DC: American Psychiatric Association, 1994.

20. Reisberg B. Functional assessment staging (FAST). Psychopharmacol Bull 1988;24:653-9.

21. Charlson ME. Studies of prognosis: progress and pitfalls. J Gen Intern Med 1987;2:359-61.

22. Waterlow J. Pressure sores: a risk assessment card. Nurs Times 1985;81:49-55.

23. Volicer L, Hurley AC, Lathi DC, et al. Measurement of severity in advanced Alzheimer's disease. J Gerontol 1994;49:M223-6.

24. Cummings JL, Mega M, Gray K, et al. The neuropsychiatric inventory: comprehensive assessment of psychopathology in dementia. Neurology 1994;44:2308-14.

25. Cohen-Mansfield J, Marx MS, Rosenthal AS. A description of agitation in a nursing home. J Gerontol 1989;44:M77-84.
26. Zwakhalen SM, Hamers JP, bu-Saad HH, et al. Pain in elderly people with severe dementia: a systematic review of behavioural pain assessment tools. BMC Geriatr 2006;6:3.

27. Kiely DK, Volicer L, Teno J, et al. The validity and reliability of scales for the evaluation of end-of-life care in advanced dementia. Alzheimer Dis Assoc Disord 2006;20:176-81.

28. Weiner MF, Martin-Cook K, Svetlik DA, et al. The quality of life in late-stage dementia (QUALID) scale. J Am Med Dir Assoc 2000;1:114-16.

29. Wimo A, Winblad B. Resource utilisation in dementia: RUD Lite. Brain Aging 2003;3:48-59.

30. Zarit SH, Reever KE, Bach-Peterson J. Relatives of the impaired elderly: correlates of feelings of burden. Gerontologist 1980;20:649-55.

31. Zigmond AS, Snaith RP. The hospital anxiety and depression scale. Acta Psychiatr Scand 1983;67:361-70.

32. Herdman M, Gudex C, Lloyd A, et al. Development and preliminary testing of the New five-level version of EQ-5D (EQ-5D-5L). Qual Life Res 2011;20:1727-36.

33. Engel SE, Kiely DK, Mitchell SL. Satisfaction with end-of-life care for nursing home residents with advanced dementia. J Am Geriatr Soc 2006;54:1567-72.

34. Mitchell SL, Kiely DK, Jones R, et al. Advanced dementia research in the nursing home: the CASCADE study. Alzheimer Dis Assoc Disord 2006;20:166-75.

35. Sampson EL, Jones L, Thune-Boyle IC, et al. Palliative assessment and advance care planning in severe dementia: an exploratory randomized controlled trial of a complex intervention. Palliat Med 2011;25:197-209.

36. Gold Standards Framework. The gold standards framework. NHS End of Life Care Programme, 2008. http://www. goldstandardsframework.org.uk/ (accessed Apr 2014).

37. Thuné-Boyle ICV, Sampson EL, Jones L, et al. Challenges to improving end of life care of people with advanced dementia in the UK. Dementia 2010;9:285-98.

38. Walsh K, Jones L, Tookman A, et al. Reducing emotional distress in people caring for patients receiving specialist palliative care. Randomised trial. Br J Psychiatry 2007;190:142-7.

39. Braun V, Clarke V. Using thematic analysis in psychology . Qual Res Psychol 2006;3:77-101.

40. Spencer L, Ritchie J, Lewis J, et al. Quality in qualitative evaluation: a framework for assessing research evidence. London: The Cabinet Office, 2003. http://www.civilservice.gov.uk/wp-content/uploads/ 2011/09/a_quality_framework_tcm6-38740.pdf (accessed May 2014)

41. Craig N, Dieppe $P$, Macintyre S, et al. Developing and evaluating complex interventions: the new Medical Research Council guidance. BMJ 2008;337:a1655.

42. Michie S, West R. Moving from theory to practice and back in social and health psychology. Health Psychol 2013;32: 581-5

43. Chambers DA, Glasgow RE, Stange KC. The dynamic sustainability framework: addressing the paradox of sustainment amid ongoing change. Implement Sci 2013;8:117.

44. Pawson R, Tilley N. Realistic evaluation. London: Sage Publications, 1997.

45. Candy B, Jones L, Drake R, et al. Interventions for supporting informal caregivers of patients in the terminal phase of a disease. Cochrane Database Syst Rev 2011;(6):CD007617.

46. The MARQUE programme. http://www.esrc.ac.uk/myesrc/grants/ES L001780.1/read (accessed May 2014). 


\section{Correction}

Elliott M, Harrington J, Moore K, et al. A protocol for an exploratory phase I mixed-methods study of enhanced integrated care for care home residents with advanced dementia: the Compassion Intervention. BMJ Open 2014;4:e05661. The Acknowledgements section of this paper should be:

Acknowledgements We are grateful for the significant scientific input of Michael King, Stephen Morris, Irwin Nazareth, and Rumana Omar from University College London and Gerard Leavey from the University of Ulster. All were co-applicants on the funding proposal for the Compassion Programme and have continued to provide expert advice throughout the programme. In particular they have all provided expertise in the development and evaluation of complex healthcare interventions. Specific contributions have been made to the conduct of the following elements: quantitative studies (MK and IN), health economics $(\mathrm{SM})$, statistics (RO) and qualitative studies (GL).

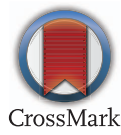

BMJ Open 2014;4:e005661. doi:10.1136/bmjopen-2014-005661corr1 